\title{
Geriatric Patients and Symptomatic Urinary Tract Infections: Analysis of Bacterial Range and Resistance Rates at a 3rd Level of Care Hospital in Germany
}

\author{
Ann-Sera Manseck ${ }^{a}$ Wolfgang Otto ${ }^{a, b, c}$ Marco Schnabel $^{a}$ Stefan Denzinger ${ }^{a}$ \\ Maximilian Burger ${ }^{a}$ Philipp Julian Spachmann ${ }^{a}$ \\ aDepartment of Urology Caritas St. Josef Medical Center, University of Regensburg, Regensburg, Germany; \\ bUrologie im Gesundheitsforum, Regensburg, Germany; ' Nierenzentrum Eichstätt, Ambulanz für Urologie, Eichstätt, \\ Germany
}

\begin{abstract}
Keywords
Antibiotic stewardship - Antimicrobial resistance rates .

Calculated antibiotic therapy · Community-acquired urinary tract infection - Elderly patients · Geriatric patients . Infection · Symptomatic urinary tract infection · Urinary tract infection
\end{abstract}

\begin{abstract}
Background: Increasing life expectancy and demographic change result in a rising number of geriatric patients, but knowledge about geriatric treatment options and adapted diagnostic pathways is low. Simultaneously, urinary tract infections (UTIS) are common in older people and often difficult to treat. Methods: Patients with a UTI at a urological outpatient department between January 01, 2016, and December 31, 2017, were retrospectively identified in the data system. One thousand one hundred fifteen patients with "N30" and "N39" diagnosis were included in this study, 965 of which were under the age of 75 (group 1) and 150 of which 75 years or older (group 2). Results: Of 150 patients in group $2,41.6 \%$ had nitrite-positive urine (vs. $20.9 \%$ in group 1, $p<$ 0.05 in $x^{2}$ testing). Patients in group 2 often had indwelling devices ( 30.3 vs. $6.0 \%, p<0.05)$ and presented a sterile urine
\end{abstract}

karger@karger.com www.karger.com/uin

Karger $\stackrel{\text { ' }}{5}$

GOPEN ACCESS (c) 2021 The Author(s).

Published by S. Karger AG, Basel

This is an Open Access article licensed under the Creative Commons Attribution-NonCommercial-4.0 International License (CC BY-NC) (http://www.karger.com/Services/OpenAccessLicense), applicable to the online version of the article only. Usage and distribution for commercial purposes requires written permission. culture in only $20.7 \%$ (vs. $40.1 \%$ in group 1). Regarding calculated antibiotic therapy, there were significant differences concerning prescription of ciprofloxacin (34.9 vs. $25 \%, p<$ $0.05)$ and fosfomycin (12.7 vs. $40.7 \%, p<0.05)$. Conclusion: UTI in geriatric patients should be treated differently than in younger patients because antibiotic resistance is high. The group with indwelling devices is complex. Individual strategies for geriatric patients should be considered.

(C) 2021 The Author(s).

Published by S. Karger AG, Basel

\section{Background}

Urinary tract infection (UTI) is a common disease in urological everyday healthcare. Incidence rises in patients over the age of 65 in comparison with patients of middle age, where UTI incidence is the lowest. Prevalence of asymptomatic bacteriuria (ASB) also rises in the elderly [1, 2]. This development correlates with geriatric patients also needing urinary catheters more often or suffering from urinary incontinence, which makes them more likely to have UTIs $[3,4]$. Increased susceptibility to UTIs also arises from functional conversions in the urinary 
tract in old age and the growing number of comorbidities $[2,5,6]$. Furthermore, the fact whether a patient has had UTIs before correlates with increased residual urine in the bladder in female patients [7]. After all, $16 \%$ of hospital admissions in patients over the age of 65 are due to UTIs [8].

Additionally, incidence of UTIs in the elderly varies according to their living situation: in patients living selfsufficiently at home, only half as many UTI cases occur as compared to nursing home patients $[9,10]$. Diagnosing UTI in geriatric patients is sometimes difficult. Patients in need of care, cognitively or communicatively impaired, and with long-persistent UTI symptoms cannot be diagnosed and treated the same way as other patients to avoid overtreatment. Relying wholly on personal anamnesis in these cases proves to be insufficient, whereas relying completely on urine analysis results seems inadequate as well, due to the high prevalence of ASB. There have been attempts to install geriatric diagnosing tools for UTI to resolve this dilemma. These combine the assessment of UTI symptoms, unspecific symptoms in geriatric patients like changes of behavior and mental status, and urine testing [11].

These difficulties in diagnosing UTI in the elderly explain frequent hospital admissions due to UTI exacerbation, as UTIs are easily overlooked and therefore allowed to progress untreated - with sometimes grave consequences in frail patients [9]. On the other hand, due to unspecific symptoms, overdiagnosis and overtreatment of UTI in geriatric patients is common as well [12].

Furthermore, elderly and frail patients often find it physically difficult to follow the procedure of obtaining a midstream urine sample. Therefore, contaminated urine samples are common in geriatric patients, complicating or falsifying the results of required diagnostic tests. Typical symptoms of UTI in older patients are frequently replaced by a variety of unspecific ones. Dysuria, fever, and leukocyturia are often missing; instead, patients show, for example, loss of appetite, coughing, or disorientation. Moreover, UTI in geriatric patients seems to be caused by a dissimilar range of bacteria, particularly biofilm-producing germs [13].

Concerning therapeutic options for UTI in geriatric patients can be challenging, but there are few differences compared to younger patient groups [14]. They mainly concern adapting dosages in antibiotics to match pharmacodynamics and -kinetics in the elderly. For example, drug absorption in the gastrointestinal tract is lower as well as compliance in drug intake; in addition, comorbid patients often take a variety of drugs simultaneously, which emphasizes the need to check for possible drug interactions. Diminished renal function and the possibility of a concurrent second bacterial infection with a different bacterial range must be taken into consideration whilst choosing an appropriate antibiotic. For nonantibiotic UTI prophylaxis, the use of cranberries or D-mannose as well as locally substituting estrogen is discussed $[1,15$, 16].

Different types of UTIs can be differentiated: positive urine culture with urinary tract pathogens in patients without complaints is called ASB. The health care-associated urinary tract infection is a UTI in hospitalized patients, and the catheter-associated urinary tract infection is a UTI in patients with indwelling urinary tract catheters. The aim of this study was to investigate if geriatric patients are at a special treatment situation due to their age in differences of bacterial species and antimicrobial resistance.

\section{Methods}

Study Setting and Design

A retrospective investigation on bacterial range, antibiotic resistance rates, and calculated therapy concerning geriatric patients was conducted at the Caritas St. Josef Medical Centre Regensburg (CSJR) urology emergency reception. CSJR serves as a tertiary referral hospital for Regensburg and its surrounding regions in north-eastern Bavaria, Germany; its urology department represents around 60 inpatient beds and a yearly number of cases of around 4,300 and is also the Department of Urology of the University of Regensburg. Geriatric patients with symptomatic UTI were compared to patients under the age of 75 with symptomatic UTI.

\section{Patients and Variables}

Patients who were admitted to the urological emergency reception due to symptomatic UTI between January 01, 2016, and December 31,2017, were retrospectively identified in the clinic-wide documentation system. One thousand one hundred fifteen patients with "N30" and "N39" diagnosis following ICD10 criteria were included in this study, 965 of which were under the age of 75 (group 1) and 150 over 75 years old (group 2). Four hundred eighty-four patients were excluded who were either underage or had no urine culture performed. Parameters retrieved from the patient record included the following: (i) demographics; (ii) recent antibiotic treatments in the last 30 days; (iii) pre-existing risk factors, for example, whether the patient was living at a nursing home facility prior to admission or indwelling devices like urinary catheters at time of urine testing; (iv) calculated therapy prescribed at emergency reception.

\section{Microbiology}

Required diagnostic urine testing was performed at the CSJR branch of SYNLAB Medizinisches Versorgungszentrum Weiden GmbH according to DIN EN ISO 15189:2014 standards. 
Table 1. Calculated therapy for patients with UTI aged under 75 compared to patients aged $\geq 75$ from CSJR hospital from January 2016 to December $2017^{\text {a }}$

\begin{tabular}{lccc}
\hline Parameter & \multicolumn{2}{l}{ Results } & \\
\cline { 2 - 4 } & group 1 $(n=860)$ & group 2 $(n=126)$ & $p$ value \\
\hline Ciprofloxacin prescribed, $n(\%)$ & $\mathbf{2 1 5 ( 2 5 )}$ & $\mathbf{4 4}(\mathbf{3 4 . 9})$ & $<\mathbf{0 . 0 0 1}$ \\
Other fluoroquinolones prescribed, $n(\%)$ & $5(0.6)$ & $0(0)$ & na \\
Trimethoprim-sulfamethoxazole prescribed, $n(\%)$ & $23(2.7)$ & $6(4.8)$ & 0.20 \\
Amoxicillin prescribed, $n$ (\%) & $46(5.4)$ & $11(8.7)$ & 0.13 \\
Amoxicillin-clavulanic acid prescribed, $n(\%)$ & $154(17.9)$ & $31(24.6)$ & 0.07 \\
Cefuroxime prescribed, $n(\%)$ & $60(7.0)$ & $11(8.7)$ & 0.48 \\
Fosfomycin prescribed, $n(\%)$ & $350(\mathbf{4 0 . 7})$ & $\mathbf{1 6 ( 1 2 . 7 )}$ & $<\mathbf{0 0 1}$ \\
Nitrofurantoin prescribed, $n(\%)$ & $2(0.2)$ & $1(0.8)$ & na \\
Nitroxoline prescribed, $n(\%)$ & $2(0.2)$ & $0(0)$ & na \\
Other antibiotic substances prescribed, $n(\%)$ & $3(0.3)$ & $6(4.8)$ & na \\
\hline
\end{tabular}

Boldface indicates statistically significant difference between groups $(p<0.05)$. Group 1: patients aged $<75$. Group 2: patients aged $\geq 75$. UTI, urinary tract infection; na, nonapplicable. ${ }^{a}$ All percentages shown represent patients for whom data were available (i.e., excluding the missing cases).

\section{Statistical Analysis}

The study database was created using Microsoft Excel 2019; all analyses were performed using IBM SPSS statistics 25.0 software, utilizing the $\chi^{2}$ test for categorical variables and the Mann-Whitney $U$ test for continuous variables. All test results with a $p$ value of $<0.05$ were considered statistically significant. Throughout the text, each of the percentages displayed represents the "valid percentage," which indicates the percentage excluding the missing data from the denominator.

\section{Results}

A total of 1,115 patients with symptomatic UTI who matched the inclusion criteria were identified during the study period. This patient group consisted of 262 male (23.5\%) and 853 female (76.5\%) patients with a mean age of 46.5 years ( $95 \%$ confidence interval $45.27-47.75$; range 18-97; median 43.0). A total of 150 patients over the age of 75 were identified, "group 2," comprising $13.5 \%$ of the overall patient cohort, and a total of 965 patients of 74 years and below, "group 1." Patients in group 1 showed nitrite-positive urine results in 192 cases (20.9\%), in contrast to 57 patients in group $2(41.6 \%, p<0.05)$. Seven hundred seventy-three $(82.1 \%)$ patients in group 1 had no antimicrobials prescribed in the last 30 days prior to the day of examination versus 111 (78.7\%) in group 2. No statistically significant differences were found for previous antibiotic therapy within 30 days. Fifty-seven (6.0\%) patients had a documented indwelling device in the urinary tract (e.g., urinary catheter) on the day of examina- tion in group 1 and $44(30.3 \%)$ patients in group $2(p<$ 0.05).

Calculated antibiotic therapy was prescribed in 860 cases $(91.9 \%)$ of group 1 and in 126 cases (88.7\%) of group 2. Of these cases, in $25.0 \%$ of group 1, the chosen antibiotic substance was ciprofloxacin $(34.9 \%$ in group $2, p<$ 0.05 ), and in $40.7 \%$ of group 1 , the chosen antibiotic substance was fosfomycin $(12.7 \%$ in group $2, p<0.05)$. Further calculated antibiotic choices are shown in Table 1.

Urine culture testing was found to be sterile in 20.7\% of group 2 and in $40.1 \%$ of group 1 . Bacterial species isolated in urine cultures for groups 1 and 2 are shown in Table 2. Some samples showed multiple bacterial growth in the urine culture: $12.7 \%$ of group 2 had 2 germs isolated (5.8\% of group $1, p<0.05)$, and $3.3 \%$ of group 2 showed 3 isolated germs $(0.4 \%$ in group $1, p<0.05)$. In total, 773 isolates were found in 697 patients, 628 of these were found in 578 patients (59.9\%) of group 1 and 145 of these germs in 119 patients (79.3\%) of group 1 (see also Table 3).

Analyses on bacterial range and antibiotic resistance rates refer to these numbers. The most detected pathogen proved to be E. coli, both in geriatric patients and in the younger patients of group 1, though to a lesser extent in group 2 (49.7 vs. $72.6 \%$ ). Some bacterial pathogens were more frequently seen in geriatric patients than in group 1, such as Klebsiella spp., Proteus spp., and Pseudomonas aeruginosa. Interestingly, the amount of multiresistant bacteria is low: no MRSA were found, and 3 MRGN was isolated in 22 cultures of nongeriatric patients (3.5\%) and 
Table 2. Bacterial species isolated from urine cultures of patients with UTI aged under 75 compared to patients aged $\geq 75$ from CSJR hospital from January 2016 to December $2017^{\mathrm{a}}$

\begin{tabular}{lccc}
\hline Parameter & \multicolumn{2}{l}{ Results } & \\
\cline { 2 - 4 } & $\begin{array}{l}\text { all isolates group 1 } \\
(n=628)\end{array}$ & $\begin{array}{l}\text { all isolates group 2 } \\
(n=145)\end{array}$ & $p$ value \\
\hline Citrobacter spp. isolates, $n(\%)$ & $12(1.9)$ & $4(2.8)$ & na \\
E. coli isolates, $n$ (\%) & $\mathbf{4 5 6 ( 7 2 . 6 )}$ & $\mathbf{7 2 ( 4 9 . 7 )}$ & $<\mathbf{0 . 0 0 1}$ \\
Enterobacter spp. isolates, $n(\%)$ & $7(1.1)$ & $6(4.1)$ & na \\
Enterococcus spp. isolates, $n(\%)$ & $15(2.4)$ & $8(5.5)$ & na \\
Klebsiella spp. isolates, $n(\%)$ & $\mathbf{3 0 ( 4 . 8 )}$ & $\mathbf{1 3}(\mathbf{1 0 . 3 )}$ & $\mathbf{0 . 0 1}$ \\
Proteus spp. isolates, $n(\%)$ & $\mathbf{2 3 ( 3 . 7 )}$ & $11(7.6)$ & $\mathbf{0 . 0 1}$ \\
Pseudomonas aeruginosa isolates, $n(\%)$ & $6(1.0)$ & $9(6.2)$ & na \\
Staphylococcus spp. isolates, $n(\%)$ & $51(8.1)$ & $2(1.4)$ & na \\
Streptococcus spp. isolates, $n(\%)$ & $24(3.8)$ & $5(3.4)$ & na \\
Other isolates, $n$ (\%) & $4(0.6)$ & & \\
\hline
\end{tabular}

Boldface indicates statistically significant difference between groups $(p<0.05)$. Group 1: patients aged $<75$. Group 2: patients aged $\geq 75$. UTI, urinary tract infection; na, nonapplicable. ${ }^{a}$ All percentages shown represent patients for whom data were available (i.e., excluding the missing cases).

8 of geriatric patients $(5.5 \%)(p=0.35)$. ESBL was found in 28 nongeriatric $(4.5 \%)$ and 10 geriatric $(6.9 \%)$ patient cultures $(p=0.2)$, and no statistical significance was found (see also Table 4 ).

In vitro antimicrobial susceptibility results for the 628 germs isolated in group 1 and the 145 germs isolated in group 2 are summarized in Table 5. Bacterial species isolated from geriatric patients frequently showed resistance rates above $20 \%$ to multiple classes of antibiotics, including ciprofloxacin, trimethoprim-sulfamethoxazole, amoxicillin, amoxicillin-clavulanic acid, and cefuroxime. The resistance to these antibiotics was more common in geriatric patients than in the younger patients of group 1. Only some antibiotic substances, for example, piperacillin-tazobactam and imipenem, retained good activity below $20 \%$ resistance rates - almost all of them intravenously applied substances. Notable exception was fosfomycin, however with a resistance rate not yet up to $20 \%$ (17.2\%). Antibiotic susceptibility results when evaluating only $E$. coli isolates presented comparable results.

\section{Discussion}

This study reports on a large cohort of patients with symptomatic UTI in the emergency care of a German hospital, especially comparing geriatric and nongeriatric patients. The findings of this study confirm that UTI in geriatric patients should be treated differently than in
Table 3. Number of isolates in nongeriatric and geriatric patients

\begin{tabular}{lcclcc}
\hline Distribution & \multicolumn{2}{l}{ Nongeriatric $(n=965)$} & & \multicolumn{2}{l}{ Geriatric $(n=150)$} \\
\cline { 2 - 3 } \cline { 5 - 6 } & $n$ & $\%$ & & $n$ & $\%$ \\
\hline No isolates & 387 & 40.1 & & 31 & 20.7 \\
1 species & 531 & 55 & & 97 & 64.7 \\
2 species & 44 & 4.6 & & 18 & 12 \\
3 species & 3 & 0.3 & & 4 & 2.7 \\
\hline
\end{tabular}

Table 4. Multiresistant bacteria in nongeriatric and geriatric patients

\begin{tabular}{lrrrrr}
\hline Multiresistant bacteria & \multicolumn{2}{c}{ Nongeriatric } & & \multicolumn{2}{c}{ Geriatric } \\
\cline { 2 - 3 } \cline { 6 - 7 } & $n$ & $\%$ & & $n$ & $\%$ \\
\hline 3 MRGN & & & & \\
$\quad$ No 3 MRGN & 606 & 96.5 & & 137 & 94.5 \\
$\quad 3$ MRGN & 22 & 3.5 & & 8 & 5.5 \\
MRSA & 628 & 100.0 & & 145 & 100.0 \\
$\quad$ No MRSA & 0 & 0 & & 0 & 0 \\
MRSA & & & & & \\
ESBL & 600 & 95.5 & & 135 & 93.1 \\
$\quad$ No ESBL & 28 & 4.5 & & 10 & 6.9 \\
$\quad$ ESBL & & & &
\end{tabular}


Table 5. Antibiotic susceptibility for germs isolated from urine cultures of patients with UTI aged under 75 compared to patients aged $\geq 75$ from CSJR hospital from January 2016 to December $2017^{a}$

\begin{tabular}{|c|c|c|c|c|c|}
\hline \multirow[t]{2}{*}{ Parameter } & \multicolumn{5}{|l|}{ Results } \\
\hline & $\begin{array}{l}\text { all isolates } \\
\text { group } 1 \\
(n=628)\end{array}$ & $\begin{array}{l}\text { all isolates } \\
\text { group } 2 \\
(n=145)\end{array}$ & $p$ value & $\begin{array}{l}\text { E. coli isolates } \\
\text { group } 1 \\
(n=456)\end{array}$ & $\begin{array}{l}E \text {. coli isolates } \\
\text { group } 2 \\
(n=72)\end{array}$ \\
\hline Ciprofloxacin-resistant isolates, $n(\%)$ & $92(14.6)$ & $38(26.2)$ & 0.02 & $55(12.1)$ & $18(25.0)$ \\
\hline Levofloxacin-resistant isolates, $n(\%)$ & $80(12.7)$ & $38(26.2)$ & $<0.001$ & $55(12.1)$ & $18(25.0)$ \\
\hline Trimethoprim-sulfamethoxazole-resistant isolates, $n(\%)$ & $122(19.4)$ & $41(28.3)$ & 0.026 & $97(21.3)$ & $18(25.0)$ \\
\hline Penicillin-resistant isolates, $n(\%)$ & $597(95.1)$ & $142(97.9)$ & na & $456(100.0)$ & $72(100)$ \\
\hline Oxacillin-resistant isolates, $n(\%)$ & $579(92.2)$ & $136(93.8)$ & na & $456(100.0)$ & $72(100)$ \\
\hline Erythromycin-resistant isolates, $n(\%)$ & $570(90.8)$ & $134(92.4)$ & na & $454(99.6)$ & $72(100)$ \\
\hline Amoxicillin-resistant isolates, $n(\%)$ & $294(46.8)$ & $88(60.7)$ & na & $181(39.7)$ & $33(45.8)$ \\
\hline Amoxicillin-clavulanic acid-resistant isolates, $n(\%)$ & $125(19.9)$ & $59(40.7)$ & na & $94(20.6)$ & $23(31.9)$ \\
\hline Ampicillin-sulbactam-resistant isolates, $n(\%)$ & $129(20.5)$ & $59(40.7)$ & na & $97(21.3)$ & $23(31.9)$ \\
\hline Cefazolin-resistant isolates, $n(\%)$ & $546(86.9)$ & $133(91.7)$ & na & $449(98.5)$ & $72(100)$ \\
\hline Cefuroxime-axetil-resistant isolates, $n(\%)$ & $81(12.9)$ & $51(35.2)$ & na & $34(7.5)$ & $9(12.5)$ \\
\hline Cefuroxime-cefotiam-resistant isolates, $n(\%)$ & $79(12.6)$ & $50(34.5)$ & na & $32(7.0)$ & $9(12.5)$ \\
\hline Cefotaxime-resistant isolates, $n(\%)$ & $56(8.9)$ & $29(20.0)$ & na & $27(5.9)$ & $6(8.3)$ \\
\hline Ceftriaxone-resistant isolates, $n(\%)$ & $55(8.8)$ & $29(20.0)$ & na & $26(5.7)$ & $6(8.3)$ \\
\hline Ceftazidime-resistant isolates, $n(\%)$ & $121(19.3)$ & $28(19.3)$ & na & $26(5.7)$ & $5(6.9)$ \\
\hline Gentamicin-resistant isolates, $n(\%)$ & $69(11)$ & $21(14.5)$ & na & $25(5.5)$ & $6(8.3)$ \\
\hline Piperacillin-resistant isolates, $n(\%)$ & $261(41.6)$ & $60(41.4)$ & na & $176(38.6)$ & $32(44.4)$ \\
\hline Piperacillin-tazobactam-resistant isolates, $n(\%)$ & $43(6.8)$ & $11(7.6)$ & na & $32(7.0)$ & $7(9.7)$ \\
\hline Imipenem-resistant isolates, $n(\%)$ & $6(1)$ & $1(0.7)$ & 0.002 & $1(0.2)$ & $0(0)$ \\
\hline Meropenem-resistant isolates, $n(\%)$ & $20(3.2)$ & $9(6.2)$ & na & $2(0.4)$ & $0(0)$ \\
\hline Fosfomycin-resistant isolates, $n(\%)$ & $58(9.2)$ & $25(17.2)$ & 0.009 & $4(0.9)$ & $0(0)$ \\
\hline Teicoplanin-resistant isolates, $n(\%)$ & $536(85.4)$ & $124(85.5)$ & na & $454(99.6)$ & $72(100)$ \\
\hline Vancomycin-resistant isolates, $n(\%)$ & $536(85.4)$ & $125(86.2)$ & na & $454(99.6)$ & $72(100)$ \\
\hline Linezolid-resistant isolates, $n(\%)$ & $536(85.4)$ & $125(86.2)$ & na & $454(99.6)$ & $72(100)$ \\
\hline
\end{tabular}

Boldface indicates statistically significant difference between groups $(p<0.05)$. Group 1: patients aged $<75$. Group 2: patients aged $\geq 75$. UTI, urinary tract infection; na, nonapplicable. ${ }^{a}$ All percentages shown represent patients for whom data were available (i.e., excluding the missing cases).

younger patient groups. Multiple bacterial growths were found more commonly in geriatric patients ( 2 pathogens in $12.7 \%$ of group 2 vs. $5.8 \%$ of group $1, p<0.05$; and 3 pathogens in $3.3 \%$ of group 2 vs. $0.4 \%$ in group $1, p<$ $0.05)$. Geriatric patients differed also in bacterial range, as Klebsiella spp., Proteus spp., and Pseudomonas aerugino$s a$ were isolated in $>25 \%$ of all pathogen isolates ( 39 out of 145 isolates in group 2 with each of these pathogens $10.3,9.0$, and $7.6 \%$, respectively). These results were similar to a study performed by Thiesemann et al. [13], which also showed a deviating bacterial range in geriatric patients [11].

Of particular interest and concern, resistance rates against antibiotic substances found in isolates from geriatric patients frequently surpass $20 \%$. International and national guidelines on UTI treatment have spoken out against calculated therapy with antibiotic agents known to have a resistance rate $>20 \%$. Therefore, results of this study suggest only few options remain for calculated treatment in geriatric patients, including fosfomycin, piperacillin-tazobactam, and imipenem. Among these options remains only 1 choice that can be given orally - fosfomycin - implicating that hospital admissions for intravenous antibiotic treatment in geriatric patients could rise soon. On the other hand, fosfomycin is off label in patients with a complicated UTI as it is only labeled for use in uncomplicated UTI and women. Increasing efforts in antibiotic stewardship practices could help delay the spread of antibiotic resistance: the use of antibiotics has to be restricted only in the situation of a symptomatic UTI, but not in an ASB. Also, as a part of measures of antibiotic stewardship, strategies of calculated antibiotic treatment regimens should not only consider the local niveau of antibiotic resistance, but also the age of the treated patient. 
There are several limitations to this study, apart from its retrospective nature. Due to limited available information, not all cases included into the study provided full data from the medical charts, and therefore percentages displayed in the text are represented as "valid percentages." Also, no comorbidity data were examined. This study cohort was limited to patients from north-eastern Bavaria and might thus not be generalizable to other geographic regions.

Considering that $>20 \%$ of cases in the geriatric group showed indwelling urinary tract devices (such as urinary catheters) and received antibiotic substances in the last 30 days prior to examination, this group appears complex. Further examination of this subgroup is of need. High antibiotic resistance rates and a disparate bacterial range limit options in calculated therapy, but also individual strategies, considering reserve capacities and comorbidities, should be considered in the treatment of geriatric patients.

\section{References}

1 Mirsaidov N, Wagenlehner FM. (Urinary tract infections in the elderly). Urologe A. 2016;55(4):494-8.

2 Cortes-Penfield NW, Trautner BW, Jump RLP. Urinary tract infection and asymptomatic bacteriuria in older adults. Infect Dis Clin North Am. 2017;31(4):673-88.

3 Hazelett SE, Tsai M, Gareri M, Allen K. The association between indwelling urinary catheter use in the elderly and urinary tract infection in acute care. BMC Geriatr. 2006;6:15.

4 Girard R, Gaujard S, Pergay V, Pornon P, Martin Gaujard G, Vieux C, et al. Controlling urinary tract infections associated with intermittent bladder catheterization in geriatric hospitals. J Hosp Infect. 2015;90(3):240-7.

5 Foxman B. Epidemiology of urinary tract infections: incidence, morbidity, and economic costs. Am J Med. 2002;113(Suppl 1A):5S-13S.

\section{Statement of Ethics}

The study is exempt from ethics committee approval due to its retrospective and noninterventional character.

\section{Conflict of Interest Statement}

The authors have no conflicts of interest to declare.

\section{Funding Sources}

No funding was obtained.

\section{Author Contributions}

All authors made significant contributions to this work. W.O. was the coordinator of this study. W.O. and P.J.S. prepared the initial study concept and revised the manuscript critically. A.S.M. acquired all data and conducted statistical analysis. A.S.M. drafted the manuscript, and W.O., M.S., M.B., S.D., and P.J.S. made relevant contributions to the manuscript.
6 Yoshikawa TT. Epidemiology and unique aspects of aging and infectious diseases. Clin Infect Dis. 2000;30(6):931-3.

7 Jackson SL, Boyko EJ, Scholes D, Abraham L, Gupta K, Fihn SD. Predictors of urinary tract infection after menopause: a prospective study. Am J Med. 2004;117(12):903-11.

8 Curns AT, Holman RC, Sejvar JJ, Owings MF, Schonberger LB. Infectious disease hospitalizations among older adults in the United States from 1990 to 2002. Arch Intern Med. 2005; 165(21):2514-20.

9 Becher KF, Klempien I, Wiedemann A. (Urinary tract infections in the elderly). Z Gerontol Geriatr. 2015;48(7):588-94.

10 Nicolle LE. Urinary infections in the elderly: symptomatic of asymptomatic? Int J Antimicrob Agents. 1999;11(3-4):265-8.

11 Juthani-Mehta M, Tinetti M, Perrelli E, Towle V, Van Ness PH, Quagliarello V. Diagnostic accuracy of criteria for urinary tract infection in a cohort of nursing home residents. J Am Geriatr Soc. 2007;55(7):1072-7.
12 McMurdo ME, Gillespie ND. Urinary tract infection in old age: over-diagnosed and overtreated. Age Ageing. 2000;29(4):297-8.

13 Thiesemann R, Walter EU, Füsgen I. Nosocomial urinary tract infections in the geriatric hospital: pathogen spectrum and resistancies. Z Gerontol Geriatr. 2009;42(2):99-107.

14 Kakde P, Redkar NN, Yelale A. Urinary tract infection in elderly: clinical profile and outcome. J Assoc Physicians India. 2018;66(6): 14-7.

15 Duncan D. Alternative to antibiotics for managing asymptomatic and non-symptomatic bacteriuria in older persons: a review. Br J Community Nurs. 2019;24(3):116-9.

16 Dubbs SB, Sommerkamp SK. Evaluation and management of urinary tract infection in the emergency department. Emerg Med Clin North Am. 2019;37(4):707-23. 